> L'atteinte rénale est l'une des manifestations principales de la maladie de Fabry. Chez les hémizygotes, en l'absence de traitement, elle conduit à l'insuffisance rénale terminale, en moyenne à

\section{Atteinte rénale de la maladie de Fabry : résultats de l'enzymothérapie} Jean-Pierre Grünfeld, Laure-Hélène Noël l'âge de 40 ans. L'enzymothérapie précoce évite la progression de la maladie rénale et entraîne la disparition des dépôts lysosomaux, notamment des cellules endothéliales vasculaires rénales et glomérulaires. Cependant, quand l'enzymothérapie est débutée trop tard, à un stade où des lésions rénales cicatricielles sont déjà présentes, elle doit être associée à un traitement additionnel, comme dans les autres maladies glomérulaires. <

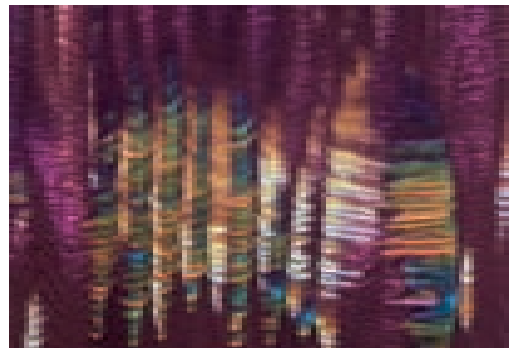

Université Paris-Descartes, Inserm U.507, AP-HP, Hôpital Necker-Enfants Malades, 149, rue de Sèvres, 75743 Paris Cedex 15, France. jean-pierre.grunfeld@ nck.ap-hop-paris.fr

\section{L'atteinte rénale de la maladie de Fabry}

L'atteinte rénale est une des manifestations principales de la maladie de Fabry. En effet, en l'absence de traitement, les hommes hémizygotes évoluent vers l'insuffisance rénale terminale (IRT), nécessitant dialyse et/ou transplantation rénale, à l'âge moyen d'environ 40 ans. La progression de l'insuffisance rénale est plus ou moins rapide selon les patients, l'IRT survenant entre la $3^{\mathrm{e}}$ et la $7^{\mathrm{e}}$ décennie [1]. Prévenir l'insuffisance rénale est donc un objectif majeur du traitement.

Pour parvenir à cet objectif, le diagnostic de maladie de Fabry doit être précoce. L'atteinte rénale se marque initialement par une protéinurie qui apparaît en moyenne entre 20 et 30 ans ; l'insuffisance rénale chronique (IRC) s'installe dans la $4^{e}$ décennie. La pression artérielle est normale ou modérément élevée. La perte annuelle de débit de filtration glomérulaire est d'environ $10 \mathrm{ml} / \mathrm{min}$ [2]. Dans certains cas, elle est plus importante et on doit alors se méfier d'une maladie glomérulaire surajoutée, y compris une glomérulonéphrite nécrosante à croissants dont plusieurs observations ont été rapportées. C'est une indication à la ponction-biopsie rénale pour reconnaître une telle association et appliquer le traitement adéquat.

Par ailleurs, la ponction-biopsie rénale peut conduire au diagnostic de maladie de Fabry. C'est le cas chez des patients protéinuriques pour lesquels le diagnostic de la maladie a été méconnu, faute d'une bonne inter- prétation des manifestations systémiques, ou chez des patients sans antécédents familiaux bien particuliers et sans manifestations extrarénales bien caractéristiques (allant jusqu'au variant «rénal » de la maladie où l'atteinte semble se limiter au rein). Les lésions histopathologiques rénales sont spécifiques. En microscopie optique, le cytoplasme des cellules glomérulaires est vacuolisé et cet aspect prédomine sur les podocytes; en coupes ultrafines, et après coloration au bleu de toluidine (Figure 1), on distingue bien les inclusions intralysosomales constituées de glycolipides qui s'accumulent faute d' $\alpha$-galactosidase A. La microscopie électronique montre bien les inclusions intralysosomales mais, en pratique, elle n'est pas indispensable au diagnostic. Les cellules tubulaires distales sont également le siège de la surcharge. Les mêmes dépôts sont présents dans les cellules des artérioles et artères intrarénales, notamment dans les cellules endothéliales et musculaires lisses. C'est l'extension des lésions vasculaires intrarénales qui est à l'origine de l'évolution de la néphropathie, entraînant une ischémie rénale progressive. Les lésions d'origine ischémique sont nonspécifiques, «cicatricielles», et elles sont détectées tôt dans le parenchyme rénal : chez 9 hémizygotes, âgés de 11 à 29 ans, ayant subi une biopsie rénale, 5 avaient déjà des lésions de glomérulosclérose, 8 des lésions tubulo-interstitielles, et 9 des lésions de nécrose des myocytes, en plus des lésions caractéristiques de la maladie de Fabry [3]. Ces lésions cicatricielles évoluent 
pour leur propre compte, persistant malgré l'enzymothérapie substitutive. Lorsque les lésions de glomérulosclérose sont étendues, elles entraînent une protéinurie abondante.

En revanche, la surcharge podocytaire n'est pas responsable de la progression vers I'IRC; elle est souvent présente chez les femmes hétérozygotes dont la grande majorité ne développera pas d'atteinte rénale cliniquement décelable. Une observation privilégiée confirme ce point de vue: le rein d'une mère hétérozygote a été transplanté à sa fille atteinte d'une glomérulonéphrite chronique idiopathique; des lésions podocytaires caractéristiques de la maladie de Fabry ont été mises en évidence pendant plus de 20 ans dans le rein transplanté [4]. La donneuse est aujourd'hui âgée de 82 ans, son rein unique est probablement le siège de lésions comparables et sa fonction rénale est normale.

Chez la plupart des femmes hétérozygotes, il n'y a pas d'atteinte rénale évolutive. Cependant, une maladie rénale évolutive peut survenir chez un petit nombre d'entre elles, du fait de l'inactivation au hasard d'un chromosome $X$; environ $12 \%$ des sujets dialysés pour une maladie de Fabry, dans les registres d'Europe et des États-Unis, sont des femmes [1].

La dialyse périodique et la transplantation rénale sont les deux traitements de suppléance rénale en cas d'IRT. Ces deux modalités de traitement s'appliquent aux patients atteints de maladie de Fabry. La survie en dialyse est moins bonne que celle des autres patients dialysés nondiabétiques, probablement du fait des complications cardio- et cérébro-vasculaires propres à la maladie de Fabry. Les registres établis en Europe et aux États-Unis fournissent des informations concordantes [1]. La survie des transplantés est identique dans la maladie de Fabry et les autres maladies; il n'y a pas de récidive du dépôt glycolipidique dans le transplant car celui-ci comporte une activité enzymatique normale. Des dépôts ont pu être observés dans des cellules circulantes provenant du receveur ou dans quelques cellules vasculaires du fait d'un microchimérisme. De façon heureuse, mais surprenante et mal expliquée, la survie des patients transplantés rénaux est identique dans la maladie de Fabry et dans les autres maladies rénales [5].

\section{Résultats de l'enzymothérapie sur l'atteinte rénale}

\section{Effets sur les cellules rénales}

On ne dispose que d'une seule étude détaillée sur les effets cellulaires rénaux après 5 à 11 mois d'enzymothérapie par agalsidase $\beta$ chez 58 patients, dont 56 hommes, ayant participé à l'étude randomisée initiale contre placebo [6]. À 5 mois, les lésions rénales sont inchangées dans le groupe placebo. En revanche, on observe une disparition complète des inclusions des cellules endothéliales, glomérulaires et vasculaires, dans le groupe agalsidase $\beta$. La clairance des dépôts est moins bonne dans les cellules musculaires lisses, moins facilement accessibles à l'enzyme apportée par voie intravasculaire. Les lésions tubulaires sont réversibles incomplètement (environ $50 \%$ ). Les lésions podocytaires sont à peine améliorées (environ $20 \%$ ) et cela n'est pas surprenant si on tient compte des observations précédentes; en outre, les podocytes sont situés sur le versant externe de la membrane basale glomérulaire, donc mal accessibles à l'enzyme circulante, et surtout leur taux de renouvellement est très faible ou nul. La surcharge podocytaire se crée très lentement sur un grand nombre d'années, même s'il existe une activité enzymatique résiduelle comme chez les hétérozygotes. Le glomérule montré sur la Figure 1 provient de la biopsie d'un hémizygote traité par algasidase $\beta$ depuis 2 ans environ; il persiste encore des inclusions podocytaires bien visibles. Il importe peu de savoir si les lésions podocytaires seront ou non complètement réversibles après plusieurs années d'enzymothérapie car, à notre point de vue, elles ne contribuent pas de façon significative à la progression de la maladie rénale.

\section{Effets cliniques}

Les effets cliniques rénaux de l'enzymothérapie sont plus difficiles à décrire à partir des essais thérapeutiques: en effet ont été inclus des patients dont la fonction rénale était initialement normale ou peu diminuée. Au terme de 30 à 36 mois de traitement, la fonction rénale, appréciée par la créatininémie, est dans l'ensemble inchangée [7]. Cependant, elle diminue légèrement chez 3 patients malgré l'enzymothérapie: il n'est pas surprenant d'apprendre que ces 3 patients étaient âgés de plus de 40 ans, qu'ils avaient une protéinurie abondante et des lésions de glomérulosclérose touchant $\geqslant 50 \%$ des glomérules sur la biopsie initiale, avant toute enzymothérapie. Ces lésions non spécifiques, post-ischémiques, se développent au fil des années, entraînant une protéinurie abondante et ne sont pas sensibles à l'enzymothérapie substitutive.

On dispose d'informations «anecdotiques» sur les effets de l'enzymothérapie chez les patients qui ont

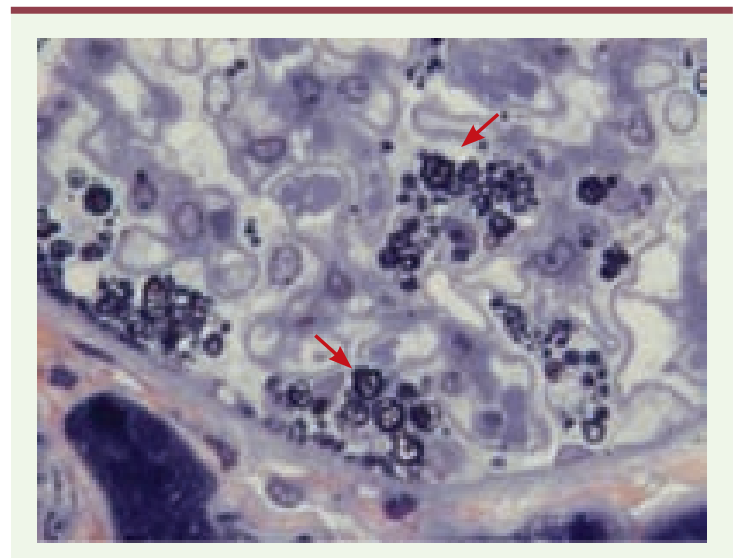

Figure 1. Glomérule d'un malade hémizygote. Les inclusions intralysosomales, lamellées (flèches rouges), sont bien visibles dans les podocytes (coupe ultrafine, coloration au bleu de toluidine). Grossissement $\times 450$. 
déjà une IRC. Souvent si l'IRC est avancée, l'enzymothérapie n'empêche pas sa progression pour les raisons indiquées plus haut. Dans d'autres cas, le traitement enzymatique ralentit la progression de I'IRC [8]; les lésions cicatricielles rénales étaient probablement encore assez limitées, permettant aux effets bénéfiques de l'enzymothérapie de s'exprimer. L'enzymothérapie a été appliquée à des patients dialysés [9]. L'évaluation des effets extrarénaux est difficile faute d'essai prospectif. La survie des patients dialysés atteints de maladie de Fabry est moins bonne que celle des autres dialysés, diabétiques exclus; on peut espérer que l'enzymothérapie améliorera cette survie, en évitant les complications vasculaires propres à la maladie de Fabry. Enfin, l'enzymothérapie a été administrée, pour les mêmes raisons, aux patients atteints de maladie de Fabry ayant subi une transplantation rénale [10].

Pour mieux répondre à certaines de ces questions, une nouvelle étude randomisée a été menée avec l'agalsidase $\beta$. Elle portait sur 82 patients dont la créatininémie était comprise entre 106 et $265 \mu \mathrm{mol} / \mathrm{l}$ ou la clairance de la créatinine était $<80 \mathrm{ml} / \mathrm{min}$ (si la créatininémie était $>106 \mu \mathrm{mol} / \mathrm{l}$ ) à l'inclusion. L'étude devait cesser lorsque 27 «événements », rénaux (augmentation de $33 \%$ de la créatininémie ou IRT) ou extrarénaux, étaient survenus. Les résultats de cette étude ne sont pas encore publiés et ne sont connus que de façon préliminaire et fragmentaire, à la suite de présentations orales. Deux informations principales ont émergé de cet essai : (1) l'enzymothérapie réduit d'environ $50 \%$ le risque de complications, mais elle ne le supprime pas dans cette population de malades traités «trop tard»; (2) l'abondance de la protéinurie est prédictive, certes des événements rénaux (comme on le sait dans de nombreuses maladies glomérulaires), mais également extrarénaux (à l'image de ce qu'on observe dans le diabète sucré).

\section{Une vue plus globale du traitement de l'atteinte rénale de la maladie de Fabry}

L'enzymothérapie débutée tôt permet de faire disparaître les dépôts glycolipidiques, notamment endothéliaux, d'éviter de nouveaux dépôts et ainsi de préserver la fonction rénale. Mais si le traitement est moins précoce, il faut tenir compte des lésions rénales non spécifiques (et probablement les évaluer par la ponction-biopsie rénale, notamment chez les sujets de plus de 30 ans, car l'expérience de l'enzymothérapie n'en est qu'à ses débuts et il faudra à l'avenir des outils et des informations pour mieux en évaluer les effets). Ces lésions relèvent d'un traitement «non spécifique», qu'on applique à toutes les maladies glomérulaires: contrôle strict de la pression artérielle $(<130 / 80 \mathrm{mmHg}$ ), diminution maximale de la protéinurie (notamment en utilisant des médicaments inhibiteurs du système rénine-angiotensine), suppression des autres facteurs susceptibles de favoriser l'évolution des lésions rénales (comme le tabagisme), etc. [11]. $\diamond$

\section{SUMMARY}

Kidney involvement in Fabry disease: results of enzyme replacement therapy

Kidney involvement is a major complication of Fabry disease. In hemizygotes, in the absence of treatment, it leads to end-stage renal disease at a mean age of 40 years. Early enzyme replacement therapy prevents progression of renal disease, and induces the clearance of lysosomal deposits, particularly from endothelial vascular and glomerular cells. However, when enzyme therapy is initiated too late, when renal scars are already present, it should be associated with additional therapy as in other glomerular diseases. $\diamond$

\section{RÉFÉRENCES}

1. Thadhani $R$, Wolf $M$, West ML, et al. Patients with Fabry disease on dialysis in the United States. Kidney Int $2002 ; 61: 249-55$.

2. Branton MH, Schiffmann R, Sabnis SG, et al. Natural history of Fabry renal disease : influence of alpha-galactosidase $A$ activity and genetic mutations on clinical course. Medicine (Baltimore) $2002 ; 81: 122-38$.

3. Gubler MC, Lenoir G, Grünfeld JP, et al. Early renal changes in hemizygous and heterozygous patients with Fabry's disease. Kidney Int 1978; 13 : 223-35.

4. Grünfeld JP, Le Porrier M, Droz D, et al. La transplantation rénale chez les sujets atteints de maladie de Fabry. Nouv Presse Med 1975 ; 29 : 2081-5.

5. 0jo A, Meier-Kriesche HU, Friedman G, et al. Excellent outcome of renal transplantation in patients with Fabry's disease. Transplantation $2000 ; 69: 2337-9$

6. Thurberg BL, Rennke H, Robert B, et al. Globotriaosylceramide accumulation in the Fabry kidney is cleared from multiple cell types after enzyme replacement therapy. Kidney Int $2002 ; 62$ : 1933-46.

7. Wilcox WR, Banikazemi M, Guffon N, et al. Long-term safty and efficacy on enzyme replacement therapy for Fabry disease. Am J Hum Genet $2004 ; 75: 65-74$.

8. De Schoenmakere G, Chauveau D, Grünfeld JP. Enzyme replacement therapy in AndersonFabry's disease : beneficial clinical effect on vital organ function. Nephrol Dial Transplant $2003 ; 18: 33-5$.

9. Kosch M, Koch HG, Oliveira JP, et al. Enzyme replacement therapy administered during hemodialysis in patients with Fabry disease. Kidney Int 2004; 66: 1279-82.

10. Mignani R, Panichi V, Giudicissi A, et al. Enzyme replacement therapy with agalsidase beta in kidney transplant patients with Fabry disease : a pilot study. Kidney Int 2004 ; 65 : 1381-5.

11. Brenner BM, Grünfeld JP. Renoprotection by enzyme replacement therapy. Curr Opin Nephrol Hypertens $2004 ; 13: 231-41$. 